\title{
Bericht über die Thätigkeit auf dermatologischem Gebiete in Budapest im Jahre 1894-5.
}

Das vergangene Jahr brachte auf dem Gebiete der Dermatologie and Syphilidologie wenig Vorträge, doch eine Fülle von Demonstrationer, welehe auf zwei Gesellschaften: dem königlichen Aerzteverein und der neugegründeten Geselischaft der Spitalsärzte - vertheilt, fast keine Woche ohne Demonstration und nachfolgender Discussion verstreichen liess. Es ist demzufolge nur mit Freude zu begrüssen, dass sich die interessirten Fachmänner unter Führung des Prof. Schwimmer entschlossen haben einen Verein der ungarischen Dermatologen in's Leben zu rufen, um solche Fragen des Fachstudiums, die bei ferner Stehenden kein volles Interesse erregen dürften, in ungestörter Musse und in eingehender Weise zu erörtern. Tnter Mitwirkung von 25 Mitgliedern wurde im Frühjahre dieses Jahres der Statutenentwurf festgestellt und liegt derselbe nunmehr dem Ministerium zur Bestätigung vor, Ich hoffe von der 'Thätigkeit des neuen Vereines bald erfreulichen Bericht liefern zu können und kehre za meiner Aufgabe der Berichterstattung über das .Jahr 1894-95 zurück.

\section{Verhandlungen des königlichen Vereins der Aerzte 1894-95.}

I. Justus: "Ueber die durch Syphilis bedingten Blutveränderungen in Hinsicht ihrer diagnostischen und therapeutischen Bedeutung." Dieser Vortrag ist seither in Virchow's Archiv, Bd. 140, in extenso erschienen, doch sei es gestattet, die Schlussergebnisse dieser Cntersuchungen für die Leser dieses Archivs in Kürze wiederzugeben. Der Yortrag enthielt das Ergebniss der Beobachtung ron 100 Syphiliskranken der Klinik des Prof. Schwimmer, sowohl vor als während und nach der specifischen Behandlung. Vortragender kam zu dem Schlusse, dass im Verhalten des Hämoglobins eine immer wiederkebrende Gesetzmässigkeit zu beobachten ist und fasst die Ergebnisse des ersten Theiles seiner Arbeit in folgende Sätze zusammen: 1. Durch die nicht behandelte Syphilis wird der Hämoglobingehalt des Blutes mehr oder weniger vermindert und diese Verminderung bleibt der Schwere der Erkrankung gemäss längere oder kürzere Zeit bestehen, um mit dern spontanen Rückgange derselben langsam zu verschwinden. 2. Wenn in 
den Stoffwechsel des syphilitisch inficirten Organismus durch Einreibung oder Injection auf einmal eine grössere (therapeutische) Quantität Quecksilber eindringt, so zeigt der Pigmentgehalt des Blutes ein bedeutendes, plötzliches Absinken (10-20\% des Fleischl'schen Hämometers). 3. Der durch Quecksilber verursachte Abfall wird je nach dem Grade der Schwere der vorhandenen Erscheinungen und dem Zustande der Ernährung bei der Schmiercur in kürzerer oder längerer Zeit ausgeglichen; bei der Injectionscur kann sich der Abfall noch nach mehreren Injectionen wiederholen, doch erscheint endlich der Zeitpunkt, wo die fortgesetzte Cur ein allmäliges Erheben des Hämoglobingehaltes bewirkt. 4. Von der Zeit an, wo der Hämoglobingehalt im Lauf der Behandlung nicht mehr sinkt, sondern zu steigen beginnt, beginnt auch die Rückbildung der vorhandenen Syphiliserscheinungen. $\breve{~}$. Der plötzliche bedeutende Abfall des Hämoglobins auf Quecksilbereinverleibung ist eine specifische nur dem Blute syphilitischer Individuen zukommende Eigenschaft und findet sich weder bei Gesunden noch bei anderweitig Erkrankten. 6. Diese specifische Reaction des Blutes ist zu constatiren von der Zeit an, wo die vom Primäraffecte entfernteren Drüsen anschwellen, bei jeder Form und Frscheinungsweise der Syphilis und verschwindet mit dem Zeitpunkte, wo die vorhandenen Erscheinungen der Syphilis den Höhepunkt ihrer Entwicklung überschreiten, um bei einer Recidive jeder Art auch wieder $z u$ erscheinen.

Justus hat nun aus diesen Sätzen gefolgert, dass das Blut bei der Syphilis specifischen Veränderungen unterliegt, dem es zeigt dem Quecksilber gegenüber ein gan\% eigenartiges, nur dem Blute von Luetikern. zukommendes Verhalten. Er musste ferner folgern, dass diese specifische Erkrankung des Blutes, welche sich durch die Reaction auf Quecksilber constatiren lässt, mit oder unmittelbar nach dem Anschwellen der entfernteren Drüsen auftretend, zeitlich der Erkrankung der anderen Organe vorangeht und endlich war es klar, dass das Verschwinden dieser specifischen Reaction, das ist die Involution der syphilitischen Blutveränderung, eintritt vor der Rückbildung der krankhaften Veränderungen in anderen Organen.

Wird die Syphilis nicht behandelt, so zeigt sich die Exolution in dem allmäligen Abfall des Hämoglobins, die Involution in dem langsamen Zunehmen desselben. Und wenn sich der Kranke einer Behandlung unterzieht, so ist der Abfall ein stärkerer, plötzlich eintretender, der Anstieg ebenfalls ein schnellerer. Die Heilung durch Quecksilber ist demzufolge von jenen Fällen, wo eine Spontanheilung (während einer gewissen Beobachtungsepoche) eintritt, nicht wesentlich, sondern nur im Bezug auf den Zeitraum verschieden.

Die Nutzanwendung auf die Diagnose ist einfach. Ueberall wo es sich um Fälle handelt, wo die Syphilis noch im floriden Stadium besteht, oder selbst in Fällen, wo eine Spätsyphilis mit nenen Herden Aer Erkrankung auftritt, in solchen Fällen, wo der Heilungsprocess noch nicht begonnen hat, ist ein Absinken des Hämoglobingehaltes, welches einige 
Stunden nach einer versuchsweise gegebenen Inunction oder Injection constatirbar ist, charakteristisch für Syphilis. Bei lange bestehenden, in Rückbildung - spontan oder in Folge einer Medication - begriffenen Symptomen ist die Probe nicht anwendbar.

An der Discussion betheiligten sich:

Havas, der unter Anerkennung der Resultate des Vortragenden dem Wunsche Ausdruck gibt, Justus möge ähnliche Untersuchungen auch bei anderen constitutionellen Krankheiten ausführen.

Schwimmer: Die Ausführungen des Vortragenden liefern eine weitere Bestätigung des Erfahrungssatzes, dass das Quecksilber auf das Virus der Syphilis einwirkt. Seine Untersuchungen zeigen, dass die Syphilis den Hämoglobininhalt herabsetzt, dass letzterer sich zu Beginn der Cur noch mehr vermindert, um nachher bei Fortführung desselben stufenweise anzusteigen. Bei anderen Krankheiten, so bei Favus, Ekzema, Lupus, Carcinom, Tuberculosis etc. zeigte die versuchsweise gegebene Quecksilbercur keine derartige Wirkung. $\mathbf{J}$ ustus wird seine Untersuchungen nach der Richtung hin nach ausdehnen, ob Jodkalium oder Arsen eine irgendwie ähnliche Einwirkung auf das syphilitische Blut auszuüben im Stande wären.

Jendrassik verfolgte mit unso grösserem Interesse die Ausführungen des Vortragenden, da er sich selbst mit der Wirkung des Quecksilbers auf das B]nt befasst hatte zur Zeit, als er die diuretische Wirkung des Calomels näher studirte. Er kann der Meinung des V. insoweit nicht zustimmen, als $V$. behauptet, dass der Abfall des Hämoglobingehaltes zu Beginn der Cur durch Zugrundegehen des Blutpigmentes bedingt sei, und dass der spätere Anstieg die Folge einer Neubildung desselben darstelle. Er hält die Hämoglobinverminderung nur für eine scheinbare, verursacht durch eine Diluition der Blutflüssigkeit. Diese wäre eine Folge der vermehrten Diffusion aus den Geweben in die Blutbahn, welche durch das im Blute kreisende Quecksilber hervorgerufen wird, wie es J endrassik in ähnlicher Weise nach Calomeldarreichung bei Hydropikern gefunden hatte. Er bestimmte bei seinen hydropischen Patienten den Wassergehalt des Blutes und fand, dass derselbe nach Darreichung von Calomel sich vermehre, jedoch späterhin bei fortgesetzter Medication wieder abnehme, um endlich ein kleinerer zu werden, als vor Beginn der Cur. Die syphilitischen Individuen seien auch meistentheils Kachektiker und könne man dieselbe Erklärung auch für diese Fälle anwenden, wenn man bedenkt, dass die Patienten im Spitale meistentheils in günstigere Ernährungsverhältnisse gelangen und wenn man ferner in Betracht nimmt, dass bei längerer Quecksilberbehandlung das Grundleiden selbst geheilt wird.

Terray stellt es als bekannte Thatsache hin, dass bei dem Fleis chl'schen Hämometer Abweichungen von 10-20 Graden innerhalb der Grenze der Beobachtungsfehler liegen. Er könne deshall den Folgerungen des V. nur mit Vorbehalt zustimmen. Um aber ein Zugrundegehen des Hämoglabins anzunehmen, wäre es unerlässlich, dass V. die Zeichen dieser 
Destruction, den Hauticterus und den Hämoglobingehalt der Faeces und des Urins nachweise.

Justus: Die Möglichkeit der Fehlerquellen bei Benützung tes Fle ischl'schen Hämometers liegt darin, dass das subjective Gefühl des Beobachters mit im Spiele ist. Dies wurde aber dadurch ausgeschlossen, dass nicht der Vortragende, sondern der untersuchte Patient - der doch vom Zwecke dieser Bestimmung keine Ahnung hatte - den Grad bestimmte, wo der rothgefürbte Keil des Hämometers und die Blutlösung dieselbe Farbe zeigten. Es liegt aber auch ganz ausserhalb der Wahrscheinlichkeit, dass man bei 2500 Bestimmungen sich eben um 10-15 Grade nur in dem Zeitpunkte getäuscht hätte, nachdem vorher eine Quecksilberinjection gemacht wurde. Wine Untersuchung des Urins und der Faeces auf Hämoglobinderivate hält auch V. für wünschenswerth, doch ist es zu weitgehend auf Icterus und Hämoglobinurie zu fahnden, da solch' schwere Symptome - wie experimentell bewiesen - sich nur einstellen nach Zerstörung eines weit grösseren Theiles des Hämoglobins, als es bei therapeutischen Quecksilber-Dosen anzunehmen berechtigt wäre. Auf die Bemerkungen Jendrassik's antwortet V., dass sich ihm bei seinen hierauf gerichteten zahlreichen Untersuchungen kein Zusammenhang zwischen der Verminderung des specifischen Gewichtes und des Pigmentgehaltes im Blute ergeben hat,

II. Marschalkó spricht äber Behandlung der Syphilis.

Fr bekräftigt unter Grundlegung einer Statistik der N e is s er'schen Klinik die Ansicht, dass die wichtigste Ursache des Tertiärismus in der fehlenden oder ungenügenden Behandlung des Frühstadiuras liege. Es soll dernzufolge ein jeder Syphilisfall vom Beginne an lange und sorgfältig behandelt werden. Die Behandlung sei aber nicht eine chablonmässige, sondern eine der Individualität des Kranken angemessene, doch solie sie mit gebührenden Pausen mindestens 2 Jahre hindurch fortgesetzt, womöglich sollte sie auch noeh im 3. Jahre nicht abgebrochen werden. Besondere Wichtigkeit sei der Behandlung den mit ganz leichten symptomen begiunenden Fällen zu schenken, da selbe gewöhnlich gar nicht oder zu kurz behandelt werden und eben deshalb das Hauptcontingent der sehweren III Syphilisfälle liefern. Unter den Behandlungsmethoden stehe obenan die Inunctionscur, dann folgen die hypodermatischen oder intramusculären Einspritzungen mit unlösbaren Ho-Salzen, hiernach diejenigen mit lösbaren Mercurverbindungen; den geringsten Werth besitzt die innerliche Medication. Das Jod hat hauptsächlich bei tertiären Erscheinungen seine Berechtigung, doch soll auch hier neben dema Jod Quecksilber verabreicht werden. Nach einigen Sätzen über die Hygiene der Syphilis bekennt sich $\mathrm{V}$. als Anhänger der Präventivbehandlung doch solle dieselbe mit Rücksicht auf die Möglichkeit des Irrthums in dor Diagnosestellung nicht als allgemein giltige Regel betrachtet werden. Eine Behandlung, die gleich nach Beginn der secundären Symptome einsetzt, sei nicht als verspätet zu betrachten und sollte man diesen Zeitpunkt bei jedem nur einigermassen zweifelhaften Falle abwarten. Bei 
Combination von Syphilis mit Tuberculose ist die chronisch intermittirende Bebandlung nicht anwendbar, dagegen eine vorsichtige Mercurialcur meistentbeils indicirt. Den Bädern komme gegenüber der Syphilis keine specifische Wirkung zu, doch seien sie oft schon frischen Symptomen gegenüber als wichtiges Hilfsmittel der Therapie zu betrachten und ganz besonders zeige sich ihre Wirkung bei inveterirten schweren Formen der sogenannten Syphilis maligna.

Havas meint, dass mit Rücksicht auf die vielen Irrthümer der Praxis ferner auf die Fälle, wo anch eine wohl ausgebildete Sclerose keine consecutiven Erscheinungen nach sich ziehe, die Behandlung nur dann zu beginnen sei, wenn die Diagnose vollständig sichergestellt ist.

Schwimmer ist auf Grund seiner Erfahrungen ein Anbänger des möglichst frübzeitigen Beginnes der Behandlung. Die Fälle, wo einer Sclerose Jreine secundäre Symptomen nachfolgten, sind so seiten, dass sie dieses allgemeine Gesetz nicht beeinträchtigen können. Die Gegner der Frühbehandlung führen an, dass dieselbe verschiedene Nervenerkrankungen verursacht, doch haben gerade die Untersuchungen, die Untersuchungen die Dr. Arthur Deutsch auf der Klinik Schwimmer's $1 \frac{1 / 2}{1 / 2}$ ahre hindurch fortgesetzt hatte, erwiesen, dass die nervösen Symptome schon vor Beginn der Quecksilberbehandlung vorhanden sind and demzufolge durch die Syphilis und nicht durch das Mercur hervorgerufen werden. Die Franzosen mit Fournier an der Spitze beansprachen 4-5 Jahre Behandlungszeit; Schwimmer meint, dies komme daher, dass die Franzosen das Quecksilber innerlich verabreichen, die energischere Inunctions- und Spritzcur dagegen äusserst selten in Anspruch nełmen. Es ist nicht nothwendig, die Kranken durch eine so lange Dauer ihrer Behandlung in Verzweiflung zu setzen, der weit überwiegende Theil heilt bei energischer intermittirender Quecksilbertherapie in $1-1 \frac{1}{2}$ Jahren. $\mathrm{Schw}$. hatte einigemale nach solcher Behandlungsdauer Reinfectionen beobachtet. Die Statistik Fournier's sei nicht zu verwerthen, da die Kranken derselben nach verschiedenen Methoden behandelt wurden.

Zur Frage sprachen noch:

Török, der seinen Zweifeln betreffs der Verlässlichkeit der vorbandenen statistischen Daten Ausdruck gibt und

Róna mit der Frage, ob V. anch die tertiären Roseolen und Papeln. in seine Statistik aufgenommen habe.

III. Feleki hält einen Vortrag über Papillomata in der Urethra, die sich sowohl in der Pars pendula nahe dem Bulbus, wie auch im Orificium Urethrae fanden. Patient hat seit 2 Jahren Blenorrhoe. V. beobachtete 5 Fälle dieser Art und war der eine Patient von Blenorrhoe verschont geblieben, was gegen die Ansicht mancher Autoren spricht, dass die Papillomen mit der Uretbralblenorrhoe in Zusammenhang stehen.

IV. Feleki. Studien aus dem Gebiete der Endoskopie. Untersuchung mittelst vergrössernden Endoskope (Megalosk op urethrale). Mit den bisher gebräuchlichen Urethroskopen sind 
manche kleine, mit freiem Auge nur schwer sichtbare Details nicht wahrzunehmen. Man kann in einer normalen Urethra die Littre'schen Drüsen, die Oeffnungen der Cowperisehen Drüsen, der Prostata und der Vesicula secuinalis nicht wahrnehmen; im entzündeten Zustande sind sie auch nicht irmmer oder nur schwer zu erkennen. So ist die wichtige periglanduläre Infiltration, die den eigentlichen Ansgangspunkt von vielen Stricturen bildet, mittelst der bisher gebräuchlichen Endoskope oftmals nicht $z \mathbf{u}$ diagnosticiren. Ebenso bilden Veränderungen in der Urethra postica oftmals die Quelle diagnostischer Irrthümer. V. hat einen kleinen Apparat construirt, mittelst dessen das endoskopische Bild 3-4mal vergrössert und genähert werden kann. Derselbe kann an jedem Endoskope leicht angebracht werden, wo die Lichtquelle an einem Punkte fixirt ist, so z. B. an Leiter'schem Panelektroskop. Das Megaloskop ist $5 \mathrm{Cm}$. lang und so leicht, dass es bei der Arbeit nicht genirt.

V. Medvei zeigt zwei Fälle von Xanthoma multiplex mit Cirrhosis hepatis hypertrophica. Der eine betrifft eine 31jährige Frau mit Xanthoma palpebrarum. Vor 8 Jahren hatte sie Schmerzen im rechten Hypochondrium und Epigastrium, ferner Gallenbrechen. Seither besteht bei ihr Dyspepsie mit manchmal auftretenden, von Fieber begleiteten Leber- und Magenschmerzen. Diese Anfälle machten den Eindruck einer Cholelithiasis, doch blieb der Icterus lange Zeit bestehen und war Leber- und Milzvergrösserung nachweisbar. Kein Ascites. Unter 5jähriger Beobachtong nahm die Leber an Umfang zu, so dass die Dämpfung jetzt von der 5. Rippe bis zur Nabelhöhe reicht; die Leber zeigt scharfe harte Ränder und eine gleichmässig gekörnte Oberfäche, die Milz ebenfalls stark vergrössert und hart, kein Ascites; der Icterus besteht auch noch heute; der Stuhl gefärbt. Patientin ist stark abgemagert. Diagnose: Hepatitis interstitialis hypertrophica. Im December v. J. ein neuer Schmerzensanfall, im Laufe dessen Pat. über heftige brennende Schmerzen auf der Beugeseite der Finger klagt; hier bilden sich parallel mit den Beugungsfurchen kleine, bis stecknadelkopfgrosse gelbe Knötchen, welche in symmetrischer Vertheilung bald in der Flachhand, und fast über allen Gelenken sich zeigen. Späterhin erscheinen selbe auch über den Streckseiten. Jetzt sind sie in dreierlei Form zu sehen: Flach an den Beugeseiten, kugelig auf den Streckseiten und zu Streifen zusammenfliessend in den grösseren Hautfurchen.

Der andere Fall betrifft einen 34jährigen Mann. Krankheitsverlauf und Symptome sind fast genau dieselben.

Beide Patienten haben in ihrer Familie mehrere Fälle von Xanthoma multiplex. Fs entsteht die Frage, ob die hypertr. Cirrhosis hepatis nur eine zufällige Complication des Xanthoma multiplex darstelle oder, wie V. meint, ihr Entstehen anf einer ähnlichen bistologischen Veränderung begründet sei.

Török führt aus, dass bei einer bedeutenden Anzahl Jer Xanthoma mult. Fälle die Her'edität nachweisbar ist. Er hält das Leberleiden der demonstrirten Fälle nicht für einfache Hepatitis interodit. hypertr. und 
bemerkt, dass in der Literatur 3 Xanthomfälle mit Leberhypertrophie bescbrieben sind und in zweien derselben Xantbomata in den Gallengängen gefunden wurden.

\section{Verhandlungen des Vereines der Spitalsarzte.}

I. Schwimmer stellt drei Kranke, die an sebwerer Syphilis leiden, vor und nimmt Anlass bei Demonstration derselben solche Fragen zu erörtern, welche im Allgemeinen nicht die gebührende Berücksichtigung gefunden haben. Erstens, dass die seit Ricord gebräuchliche Eintheilung der Syphilis in ein primäres, secundäres und tertiäres Stadium der Nosologie dieses Leidens nicht immer entspricht, denn es zeigen sich oft Erscheinungen, welche nach diesem Schema dem Tertiärismus angehören, schon zum Beginne der Krankheit, dagegen treten nicht selten zur Zeit der Späterscheinungen solche Symptome - z. B. die Roseola auf, welche zur Frühperiode gezählt werden. Darum wäre es vielleicht besser, statt von secundärer und tertiärer Syphilis, von leichter und schwerer Syphilis zu sprechen und statt Früh- und Spätsymptomen milde und schwere zu unterscheiden. Zweitens dass man sehr oft ausserordentlich schwere Erscheinungen beobachtet bei solchen Syphilitikern, denen keine entsprechende antiluetische Behandlung za Theil wurde. V. ist Anhänger der Präventivbehandlung und er beginnt dieselbe, sobald die syphilitische Affection (Primärerkrankung und nachweisbare Drüsenschwellung) constatirt ist.

Die vorgestellten Fälle betrafen:

a) Einen 27jährigen Schuhmacher; derselbe hatte im April 1892 einen Schanker. 2 Monate später traten bei ihm rothe Flecken auf, aus denen sich bald Gummata entwickeiten, die schnell zerfallend sich zu von Rupiaborken bedeckten Ulcerationen verwandelten. Der Kranke wurde keiner mercuriellen Cur theilhaftig, bis er im August 1892 auf die dermatologische Klinik aufgenommen wurde, wo ihn V, mit Decocten und intramusculären Einspritzungen von Hydrarg. sozojod. behandelte. Im Juni 1893 wurde Patient entlassen, doch kam er schon nach 4 Wochen mit neven Ulcerationen zur Aufnahme; Patient steht seit dieser Zeit in regelmässiger Behandlung and wird demnächsi die Klinik verlassen können.

b) Der zweite Kranke ist ein 35jähriger Gemeindenotar, der schon in seinem 13. Jahre durch einen Stubengenossen angesteckt wurde. Mit 20 Jahren hatte er Drüsenentzündungen, Ozaena und verlor einen Theil des Gaumondaches. Vor 9 Jahren kam er zuerst auf die Klinik, bis dahin wurde er mit $\mathrm{Hg}$ nicht behandelt. Er bekam damals 4 Monate hindurch Jod und Eisen, späterhin hatte er mehrmals recidivirende Naseneiterung, verlor das Septum narium und einen Theil der Nasenknochen. Ferner trat eine Periostitis, die zu Fistelgängen fübrte, an beiden Tibien auf Patient ist seit November in der Klinik und weist sein Zustand nach 25 Inunctionen eine sehr bedeutende Besserung auf.

c) Der dritte Fall bezieht sich auf eine 30jährige Frauensperson, die von einer primären Veränderung nichts zu sagen weiss. Vor $8 \mathrm{Mo-}$ 
naten bemerkte sie einen auf dem Körper zerstreuten rothen Ausschlag, dessen einzelne Papeln sich bald vergrösserten und zerfielen. Vor 3 Monaten liess sie sich auf die Klinik aufnehmen und waren bei ihr eine Angina specifica und am Körper weitverbreitete, von Rupiaborken bedeckte Ulcerationen constatirbar. Vor der Aufnahme machte sie eine Schmiercur durch und nahm innerlich Hg-Pillen. Ihr Zustand hat sich seit ihrem Spitalsaufenthalte unter Jod und Mercurbehandlung bedeatend gebessert.

An der Discussion nahmen Theil:

Ròna ist der Ansicht, dass die leichte und schwere Form nicht dem entspreche, was man mit secundärem und tertiärem Stadiuro ausdrücken möchte. Leichte und sehwere Formen zeigt jede Krankheit und wir müssen die Fälle, wo sich tertiäre Erscheinungen schon in der Frühperiode zeigen, als abnorme betrachten. Die drei Stadien unserer heutigen Auffassung entsprechen nicht derjenigen Ricord's.

Schwimmer erwidert, dass die althergebrachte Eintheilung sehematisch wohl sehr bequem und leicht fasslich ist, nosologisch beurtheilt jedoch sehr häufig sich als unrichtig erweist.

II. Hochhalt: Ein Fall von Lungensyphilis. Eine 26jăhrige Kranke betreffend, in deren Familie kein Fall von Tuberculose vorgelkommen und die mit Zeichen der Infiltration im linken unteren Lungenlappen und in der rechten Lungenspitze, ausserdem mit syphilitischen Hautulcerationen behaftet aufgenommen wurde. Nach 6wöchentlicher Mercurbehandlung waren die Ulcerationen geheilt and Lungenveränderungen nicht mehr nachweisbar.

Schwimmer sah mehrere Fälle von Isungensyphilis. Einmal trat selbe als Bronchopneumonia chronica auf und nahm er nur damals einen syphilitischen Ursprung derselben an, als auch Hauterscheinungen zu Tage traten. Ferner beobachtete er eine Pneumonia eatarrhalis luetischer Herkunft, welche durch Pilocarpin-Injectionen geheilt wurde. Es ist daher nicht nur Quecksilber, sondern auch eine andere resorptionsbefördernde Bebandiung von guter Wirkung.

IIJ. Rona demonstrirt eine Anzahl mit syphilitiscben Leucodermata behafteten Patientinen. Ferner eine Patientin mit primärer Affection auf der Gingiva und endlich einen Fall eines primären gangränösen Erysipels des Penis und des Hodensackes.

IV. Schwimmer. a) Urticaria ha e morrhagica. 0. F., 48jähriger Maschinenschlosser von kräftiger Constitution. Sein Leiden begann vor 7 Jahren mit noch gegenwärtig anbaltenden Jucken. Die Efflorescenzen. zeigten schon damals ihr jetziges Ausseben.

Status praesens: Au den inneren Organen keine Veränderung. Auf der ganzen Körperoberfläche sind folgende Erscheinungen zu beobachten: Neben zahlreichen hanfkorn- bis linsengrossen dunkelrothen Erhebungen sind zablreiche mehr weniger scharf begrenzte hell-braunrothe Flecken wahrzunehmen. Ausser diesen leichteren Symptomen sieht man auf den Fxtremitäten zahlreiche, die Hantoberfläche ïberragende, ein 
dunkelrothes Centrum aufweisende, hellroth begrenzte Efflorescenzen. Die Haut der Unterarme, der Ober- und Unterschenkel ist fast vollständig von ähnlichen Efflorescenzen bedeckt. Einige derselben haben ein weisses Centrum, doch einen umsomehr rothgefärbten Rand und ausserbalb letzteren einen schmalen weissen Halo. Das Symptomenbild behält seine Form unverändert bei und entspricht einer Urticaria perstans. Doch sind überdies bei dem Kranken noch solche Veränderungen zu bemerken, welche bei Nesselausschiägen überhaupt nicht zur Beobachtung kommen.' In einer Anzahl der Efflorescenzen sind umschriebene Ecchymosen zu finden, besonders ad Nates und auf der Aussenseite der Schenkel. Diese Blutaustritte sind nur in den Urticariaflecken zu beobachten, ausserhalb derselben nicht. Den allgemeinen Zustand betreffend ist noch hervorzuheben, dass Patient seit Beginn der Erkrankung an heftigen Gelenksschmerzen leidet die nur auf kurze Zeit nachlassen, besonders sind die Knie- und Schultergelenke angegriffen.

Es wären an dem Falle hervorzuheben: Die 7 Jahre lang bestehende ausgedehnte Urticaria und die seltene Erscheinung der stationären Blutaustritte innerhalb der Effiorescenzen.

Patient suchte die Klinik mehrmals auf und war auf entsprechende Behandlung und Ruhe eine Milderung des Juckens zu erreichen, doch trat dasselbe, so wie die Hämorrhagien nach Verlassen des Krankenhauses wieder heftiger auf. Der Fall bildet eine interessante Form einer angioneurotischen Hauterkrankung, da die Störung der Innervation in dem Hautgefässe eine Lähmung desselben veranlasste, die einen solch' hohen Grad erreichte, dass aus zahlreichen kleineren Gefässen Blutaustritt erfolgte

b) Lichen ruber planus atrophicans.

Patient ist ein 44jähriger Dienstmann. Sein Leiden begaun im December vergangenen Jahres. Es erschienen unter andauerndern Jucken etwa stecknadelkopfgrosse rothe Efflorescenzen, welche den grösseren Theil des Rumpfes bedeckten. Selbe waren in grösseren oder kleineren Gruppen auf den Extremitäten, besonders an der inneren Fläche des Schenkels zu finden. Die einzelnen Efflorescenzen bildeten braunrothe stecknadelkopfgrosse, zumeist mit einer centralen Delle versehene, wachsartig glänzende Pappeln, die nicht mit einander zusammenflossen. Dies kurz geschilderte Krankheitsbild war aber nur damals wabrnehmbar, als nach 2 wöchentlicher Behandlung ein universelles schuppendes Ekzem, welches dasselbe fast vollständig verdeckt hatte, verschwunden war. In der vierten Woche des Spitalaufenthaltes hatte sich das Bild bedeutend verändert. Es waren weder Gruppen noch einzelne Efflorescenzen constatirbar, auch der rothe Rand war verschwunden und es entwickelte sich allmälig der jetzt vorhandene Status. Die Stellen der sich pappelartig erhebenden Eifflorescenzen waren ausnahmslos durch kleine Einsenkungen gekennzeichnet. An den Stellen, wo die Efflorescenzen zu Gruppen gedrängt auf der entzündeten Haut sassen, hatten sich allmälig sepiabraıne Pigmentflecken eingestellt. 
c) Lichen ruber planus.

Patient, 34 Jahre alt, hatte vor 10 Jahren ein Inetisches Geschwür und 2 Jahre darauf Pappeln in der Flachiband, die auf Quecksilberpflaster verschwanden. Im September vorigen Jahres litt er an rheumatoiden Schmerzen, gegen welche ihm sein Arzt K. J. und heisse Bäder verordnete. Kurze Zeit nachher begann sein gegenwärtiges Leiden, welches in Anfange nicht besonders juckte. Es sind zur Zeit an den Extremitäten, besonders an den Beugeseiten des Unterschenkels und des Unterarmes, ferner an den Seitenregionen des Rumpfes und am Rücken vereinzelte oder zu Gruppen conglomerirte Efflorescenzen bemerkbar. Die Einzel-Efflorescenz bildet ein etwa stecknadelkopfgrosses, lichtrothes, wachsartig glänzendes Knötchen. Wo selbe zu grösseren Gruppen zusammengedrängt sind, ist ihre Farbe intensiv roth und bildet hie und da zusammenfiessende, etwa kreuzergrosse, scharf begrenzte, wenig glänzende Plaques. Auf der Hautfläche finden sich zerstreute, unregelmässigs geformte braune Pigmentflecken, deren Ränder sehr feine Schüppchen tragen.

Als Patient die Klinik zum ersten Male aufsuchte, waren die Efflorescenzen weder in so grosser Anzahl, noch so charakteristisch ausgebildet, so dass man in Hinsicht auf die Anamnese an ein syphilitisches Exanthem denken konnte. Es wurde beim Patienten mehrere Tage hindurch der Hämoglobingehalt festgestellt und hierauf eine intramusculäre Injection von $8 \mathrm{Cgr}$. Hydrargyrum sozojodolicum gegeben. Wäre das Exanthem ein syphilitisches gewesen, so hätte, da selbes noch im Fortschrciten begriffen war, ein bedeutender Abfall des Hämoglobingehaltes erfolgen müssen, wie ein solcher bei allen frisehen syphilitischen Affectionen, die auf der Klinik von Dr. Justus untersucht wurden, sich nachweisen liess. Da aber der Hämoglobingehalt sich nicht verminderte, war Syphilis in diesem Falle auszuschliessen. Der weitere Verlauf und das Auftreten von vielen neuen charakteristischen Efflorescenzen am Stamme, im Mundwinkel und am Lippenroth, ferner das bald einsetzende heftige Jucken bestätigten die Diagnose auf Lichen ruber planus. *)

V. Rona stellt einen Fall von Kra asosis vulvae (Breisky) vor. Der Fall betraf eine 49jährige Person, bei der die Innenfläche der grossen Lippen, das Vestibulum und der Damm mit einer grauweissen Hornschichte bedeckt ist. Die Nymphen sind verschwunden, das Präputium clitoridis ist hypertrophirt und mit dem linken grossen Labium verwachsen, Vestibulum und Introitus stark verengert, die Schleimhaut trocken, leicht zerreissbar, narbenartig atrophirt. Patientin klagt über unerträgliches Jucken. Sie hat vor 18 Jabren Gonorrboea und Syphilis acquirirt, die Spuren des letzteren sind noch heute bemerkbar. Der Pruritus besteht seit 6 Jahren. In der Scheide wenig Secret, keine Gonococen, im Urin nichts abnormes, Carcinoma uteri nicht rorhanden.

1) Patient ist seither unter andauendeni Arsmgebrauche vollständig genesen. 
Elischer kennt den Fall und kann mit Róna die Diagnose betreffend nicht übereinstimmen. Es handelt sich um eine Blennorrhoea chronica vetularum.

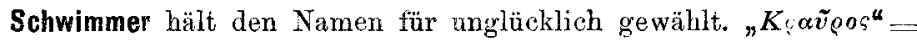
ungleichmässig, entspricht dem Zustande nicht, Atrophie wäre besser. Die weissen Plaques, welche man an der Zunge beobachten kann, finden sich aucb in der Urethra und wurden von $G \mathbf{r}$ ünfeld ${ }_{n}$ Xerosis * benannt, Breisky gebrazcht für denselben Zustand den Namen "Krauroris“. Es wäre wohl besser, ein Uebereinkommen in der Bezeichnung dieser Affection, die wir an der Schleimbaut des Mundes und der Zunge so häufig sehen, zu treffen und die Affection für eine Leukoplakie vulvae, welche die Atrophie der Epidermis verursacht hatte, anzusprechen. Die Aetiologie betreffend ist er der Ansicht, dass nur der Pruritus den ungemein grossen Juckreiz verursacht. Es liegt bein Grund vor, die Syphilis oder die Gonorrboe als cansales Moment za betrachten.

VI. Róna: Seltener Fall von Lupus vulgaris. 38jähriger Baner, dessen Lupus in seinem 10. Lebensjahre im Gesichte begann, zur Zeit den linken Lnterschenkel und die linke Kniekehle zum grösseren Theile, ferner die rechte Schulter gänzlich, zum Theile auch die linke bedeckt. Am Nacken und am Halse hat der Process die Haut zu Narbengewebe umwandelt und erstreckt sich bis zum Sternum. Die grösste Intensität exreicht die Krankheit im Gesichte. Die Nasenspitze ist weggefressen, die Ohren, die ganze linke Gesichtshälfte und die Stirn mit dicken Lupusknötchen besäet oder zu narbigen Strängen verwandelt. Am schwersten ist die linke Backe betroffen, denn hier ist eine stellenweise, mehrere $\mathrm{Cm}$. hohe Framboesie zu finden, welche stark eiternd das linke Auge bedeckt, das linke Kinn und die linke Hälfte der Lippen ungemein verdickt und verunstaltet. Auf der linken Gesichtshälfte unterscheidet sich das Bild schon von demjenigen einer lupösen Framboesie, so dass es den Gealanken wachruft, man habe es hier nicht mehr mit einem reinen Lupus, sondern mit einem auf dieser Basis entstandenen Riesencarcinom zu thun.

Justus hat auf der Klinik des Prof. Schwimmer, wo Patient längere Zeit in Beobachtung stand, den Fall beobachtet und konnte sich durch Autopsie überzeugen, dass neben dem Lupus ein grosszelliges Sarcoma alveolare vorhanden war, wie dies auch auf der dermatol. Klinik festgestellt wurde.

VII. Meisels stellt einen Fall von Atresia vulvae et strictura vestibuli vaginae vor. Dieselbe war vor 14 Jahren in Folge von Verbrühung entstanden.

VIII. Feuer einen Fall ron $\mathbb{L}_{\mathrm{i}} \mathrm{pus}$ conjunctivae, ein junges Mädchen betreffena, das auch an đer Nase Lupöse Veränderungen hat. Das linke Auge ist intact, die Comunctiva des rechten dagegen injicirt, geschwollen und verdickt. Im inneren Augenwinkel zeigt die Conjunctiva bulbi eine knotenförmige Verdickung von normaler Farbe. - Von dem oberen Conjunctivalsack hängt eine dicke Membran gleich einer doppelten 
Draperie herab, die auf beiden Seiten Knötchen zeigt. Die histologisclie Untersuchung eines exendirten Stückchens, welche $J$ ustus ausführte, ergab keinen sicheren Befund, doch hält Vortragender mit Rücksicht auf den Gesichtslupus die Diagnose Lupus conjunctivae für begründet.

Goldzieher meint, dass derartige Granulome auf der Conjunctiva nicht eben selten sind, obzwar die Lebrbücher darüber wenig enthalten. - Syphilis, Lupus, Tuberculosis sogar Scrophulose verursachen ähnliche Bildungen und oftmals kann nur die bacteriologische Untersuchung die Diagnose entscheiden.

IX. Farkas spricht über parenchymatöse Injectionen bei Leistendrüsenaffectionen nach Welander. Er hat seit August 1892142 Fälle nach dieser Methode behandelt. In allen war schon Vereiterung vorhanden. In 71 Fällen war die Injection allein genügend zur Heilung und wurde letztere durchschnittlich in $15 \frac{1}{2}$ Tagen erreicht. In den anderen 71 Fällen, wo nach der zweiten Injection der Bubo sich uicht verkleinerte und ein Eröffnen desselben nothwendig wurde, betrug die durchschnittliche Heilungsdauer $25 \cdot 3$ Tage.

$X$. Róna stellt einen Fall von Lupus erythematodes faciei, ferner einen Fall von Melanosarcoma multiplex cutis vor. Patient ist 57 Jahre alt; hatte vor 20 Jahren Malaria; seither bestehende Milzvergrösserung. Vor $4 \frac{1}{2}$ Jahren bemerkte er eine Drüsenvergrösserung in der linken Inguinalgegend; vor 1 Jahre sah er die ersten linsengrosse Knötchen in der rechten Gesässgegend; seither haben sich dieseiben ungemein vermebrt und sind jetzt in der Kreuzgegend, an den Fusszehen und in der Ellbogengegend zu sehen. - Seit einem Jahre sind die Halsdrüsen geschwollen und die Haut des Gesichtes und der Extremitaten braun verfärbt. Die Milz ist ungemein vergrössert. Die Schleimhaut der Lippen und des Mundes ist schwarzbraun. mit zerstreuten blauen Flecken. Das subjective Befinden ungestört. Patient leugnet einen Naevus gehabt zu haben, doch erscheint die Annahme eines solchen sehr begründet. Sarcoma idiopath. multipl, cutis (Kaposi) ist hier auszuschliessen.

$$
\text { Dr. Just us (Budapest). }
$$

